\title{
BMJ Open Sport and dance interventions for healthy young people (15-24 years) to promote subjective well-being: a systematic review
}

Louise Mansfield, ${ }^{1}$ Tess Kay, ${ }^{2}$ Catherine Meads, ${ }^{3}$ Lily Grigsby-Duffy, ${ }^{2}$ Jack Lane, ${ }^{4}$ Alistair John, ${ }^{2}$ Norma Daykin, ${ }^{5}$ Paul Dolan, ${ }^{6}$ Stefano Testoni, ${ }^{6}$ Guy Julier, ${ }^{4}$ Annette Payne, ${ }^{2}$ Alan Tomlinson, ${ }^{4}$ Christina Victor ${ }^{7}$

To cite: Mansfield L, Kay T, Meads C, et al. Sport and dance interventions for healthy young people (15-24 years) to promote subjective well-being: a systematic review. BMJ Open 2018;8:e020959. doi:10.1136/ bmjopen-2017-020959

- Prepublication history and additional material for this paper are available online. To view these files, please visit the journal online (http://dx.doi. org/10.1136/bmjopen-2017020959).

Received 6 December 2017 Revised 26 March 2018 Accepted 11 May 2018
Check for updates

${ }^{1}$ Department of Life Sciences, Division of Sport Health and Exercise Sciences, Brunel University London, Uxbridge, UK ${ }^{2}$ Life Sciences, Brunel University London, Uxbridge, UK

${ }^{3}$ Health, Social Care and Education, Anglia Ruskin University, Cambridge, UK ${ }^{4}$ Arts and Humanities, University of Brighton, Brighton, UK

${ }^{5}$ Health and Wellbeing, University of Winchester, Winchester, UK

${ }^{6}$ Social Policy, The London

School of Economics and

Political Science, London, UK

${ }^{7}$ Clinical Sciences, Brunel University London, Uxbridge, UK

Correspondence to

Professor Louise Mansfield;

louise.mansfield@brunel.ac.uk

\section{ABSTRACT}

Objective To review and assess effectiveness of sport and dance participation on subjective well-being outcomes among healthy young people aged 15-24 years.

Design Systematic review.

Methods We searched for studies published in any language between January 2006 and September 2016 on PsychINFO, Ovid MEDLINE, Eric, Web of Science (Arts and Humanities Citation Index, Social Science and Science Citation Index), Scopus, PILOTS, CINAHL, SPORTDiscus and International Index to Performing Arts. Additionally, we searched for unpublished (grey) literature via an online call for evidence, expert contribution, searches of key organisation websites and the British Library EThOS database, and a keyword Google search. Published studies of sport or dance interventions for healthy young people aged 15-24 years where subjective well-being was measured were included. Studies were excluded if participants were paid professionals or elite athletes, or if the intervention was clinical sport/dance therapy. Two researchers extracted data and assessed strength and quality of evidence using criteria in the What Works Centre for Wellbeing methods guide and GRADE, and using standardised reporting forms. Due to clinical heterogeneity between studies, meta-analysis was not appropriate. Grey literature in the form of final evaluation reports on empirical data relating to sport or dance interventions were included.

Results Eleven out of 6587 articles were included (7 randomised controlled trials and 1 cohort study, and 3 unpublished grey evaluation reports). Published literature suggests meditative physical activity (yoga and Baduanjin Qigong) and group-based or peer-supported sport and dance has some potential to improve subjective wellbeing. Grey literature suggests sport and dance improve subjective well-being but identify negative feelings of competency and capability. The amount and quality of published evidence on sport and dance interventions to enhance subjective well-being is low.

Conclusions Meditative activities, group and peersupported sport and dance may promote subjective wellbeing enhancement in youth. Evidence is limited. Better designed studies are needed.

Trial registration number CRD42016048745; Results.
Strengths and limitations of this study

- Prepublication of our protocol on the International Prospective Register of Systematic Reviews ensures methodological transparency and mitigates against potential post hoc decision making.

- A comprehensive research, policy and practice-relevant search strategy was used including searches of published and unpublished data, and study selection was carried out by two reviewers independently.

- Data extraction and quality assessments were conducted using standardised forms, independently by two reviewers.

- The focus on a specific target age group may have excluded evidence from studies that have aggregated data across younger and older age groups in their analysis.

- Meta-analysis was not possible due to the heterogeneity of study interventions and outcomes.

\section{INTRODUCTION}

Governments and international organisations acknowledge subjective well-being (SWB) as a policy goal. ${ }^{1-3}$ The international focus on measuring SWB is gaining recognition in some aspects of UK sport, ${ }^{4}$ dance $^{6}$ and physical activity policy. ${ }^{7}$ SWB describes well-being in terms of the good and bad feelings arising from what people do and how they think. ${ }^{8}$ Good feelings include happiness, joy, contentment and excitement while sadness, worry, stress and anxiety are examples of more negative feelings. People's experiences also involve a sense of purpose (eg, worthwhileness, meaningfulness) and pointlessness (eg, futility, boredom). Since 2011, SWB measured as satisfaction with life, worthwhileness, happiness and anxiety has been included in UK population surveys conducted by the Office of National Statistics. ${ }^{9}$ Links between sports and cultural activities 
and SWB have been reported and sport engagement is included in national-level data collection and analysis. ${ }^{10}$ Significant associations have been found between engagement in sport, the arts and enhanced SWB as measured by life satisfaction. ${ }^{11}$ Yet, it is acknowledged that SWB is a complex concept, with no single agreed definition or measure. ${ }^{12}$ The term SWB is used synonymously with a wide range of concepts including self-esteem, self-efficacy, self-determination, resilience, quality of life, mood enhancement, positive mental health, life satisfaction, worthwhileness and happiness. ${ }^{13}$ Measures of SWB use various scales that demonstrate well-being as multidimensional, for example, The Warwick and Edinburgh Mental Wellbeing Scale, ${ }^{14}$ Rosenberg's Self-Esteem Scale and ${ }^{15}$ The Profile of Mood States. ${ }^{16}$

The What Works Centre for Well-being initiative, ${ }^{17}$ funded by the Economic and Social Research Council has commissioned evidence reviews in key areas including Culture, Sport and Well-being. Following consultation with stakeholders ${ }^{18}$ four topics were identified for systematic review between 2015 and 2018 (music and singing, sport and dance, visual arts, and outdoor nature-based physical activity). This paper reports the findings of the second systematic review topic; sport and dance interventions for healthy young people (15-24 years) to promote subjective well-being.

The established definition of sport, used throughout the sector, remains that cited from the European Charter (1992) and refers to forms of physical activity either casually or formally organised in which people take part for fitness, health and well-being, social relationships or competition. ${ }^{19}$ Sport includes a wide range of individual and group activities including jogging, running, cycling, martial arts, yoga, team games and athletics. Dance is commonly defined differently from sport as a performing art form which refers to the rhythmic movements and sequences of steps usually set to music. ${ }^{20}$ Sport and dance programmes in the UK operate in different delivery, programming and funding environments, yet both sport and dance organisations identify young people as a key target group for engagement in physical activity to enhance well-being. The evidence, however, is theoretically and methodologically diverse and less attention has been given to children and adolescents. Existing evidence reviews on sport have tended to focus on physical rather than mental health or well-being outcomes ${ }^{21-23}$ or they have examined the effect of exercise in populations with specific mental health conditions such as depression ${ }^{24}$ and anxiety. ${ }^{2526}$ Dance-related reviews of evidence have examined the effectiveness of dance therapy on psychological and physical health and well-being outcomes in patients with cancer, ${ }^{27}$ for schizophrenia ${ }^{28}$ and on depression. ${ }^{29} \mathrm{~A}$ review of reviews on physical activity and mental health in children and adolescents identified an association between physical activity and positive wellbeing outcomes connected to reduced depression and anxiety, and enhanced self-esteem and cognitive function. ${ }^{30}$ No systematic review to date has focused on sport and dance interventions in healthy young people (15-24 years) to promote subjective well-being. Interventions that positively influence the well-being of young people have the potential to promote good physical and mental health. ${ }^{31-33}$ This review provides evidence that may improve understanding of the effects of sport and dance on a range of SWB measures and contribute to informing policy development, programme delivery and measurement and evaluation of sport and dance interventions to enhance well-being.

\section{METHODS}

The protocol for this systematic review was registered with the International Prospective Register of Systematic Reviews on 3 October 2016 (registration number CRD42016048745). The review follows the Preferred Reporting Items for Systematic Reviews and Meta-Analysis guidelines. ${ }^{34}$

\section{Patient and public involvement}

Participant observation by one investigator (LM) of public groups taking part in community arts and sports activities contributed to the development of the review question for this study. Patients were not involved in the conduct of the systematic review. The findings of this study will be written in accessible English and disseminated through the What Works Centre for Wellbeing website accessible by the public.

\section{Inclusion criteria}

Inclusion criteria were any comparative studies investigating any form of sport or dance compared with no sport or dance, usual routine or comparing pretest and posttest scores in healthy young people aged 15-24 years and measuring any form of subjective well-being (table 1). We included studies worldwide from countries economically similar to the UK (using OECD-DAC list of country development; http://www.oecd.org/dac/stats/daclist.htm) or with study populations similar in terms of socioeconomic status. Studies could be fully published with search dates of 2006-2016 to reflect current and long-term work on sport, dance and well-being,or grey literature (with search dates of 2013-2016). Shorter timescales for grey literature search ensured a focus on finding recent relevant studies that had not yet been published. Grey literature was included if it was a final evaluation or report on empirical data, had the evaluation of sport-related or dance interventions as the central objective and included details of authors (individuals, groups or organisations).

\section{Exclusion criteria}

Published studies were excluded if participants were paid professionals or elite athletes, or if the intervention was sport or dance therapy delivered in a clinical setting for rehabilitation purposes. We did not include studies of walking as there is existing review level evidence on the health and well-being benefits of this activity. ${ }^{35}{ }^{36}$ Grey 


\begin{tabular}{|c|c|c|}
\hline PICOS criteria & Inclusion & Exclusion \\
\hline Participants & $\begin{array}{l}\text { Participants were to be } 15-24 \text { years of age. } \\
\text { Studies from countries economically similar to the } \\
\text { UK (ie, other high-income countries with similar } \\
\text { economic systems) or with study populations that } \\
\text { have similar socioeconomic status to the UK. }\end{array}$ & $\begin{array}{l}\text { Participants with a health condition } \\
\text { diagnosed by a health professional. } \\
\text { Participants who were paid } \\
\text { professionals or elite athletes. } \\
\text { Participants in clinically based sport } \\
\text { and dance interventions. }\end{array}$ \\
\hline Intervention & $\begin{array}{l}\text { Participatory sport and dance interventions } \\
\text { including watching and performing. } \\
\text { Including sport-related and dance therapy offered } \\
\text { to enhance well-being in healthy young people. }\end{array}$ & $\begin{array}{l}\text { Clinical sport-based or dance } \\
\text { therapy. } \\
\text { Sport and dance for clinical } \\
\text { procedures such as surgery, medical } \\
\text { tests and diagnostics. } \\
\text { Walking. }\end{array}$ \\
\hline Outcomes & $\begin{array}{l}\text { Subjective well-being using any recognised } \\
\text { method or measure. }\end{array}$ & \\
\hline Study design & $\begin{array}{l}\text { Empirical research: either quantitative, qualitative } \\
\text { or mixed methods, outcomes or process } \\
\text { evaluations. } \\
\text { Grey literature: if it was a final evaluation or } \\
\text { report on empirical data, had the evaluation } \\
\text { of sport-related or dance interventions as the } \\
\text { central objective and included details of authors } \\
\text { (individuals, groups or organisations). } \\
\text { Published studies published between } 2006 \text { and } \\
2016 \text {. } \\
\text { Grey literature and practice surveys published } \\
\text { between } 2013 \text { and } 2016 \text {. }\end{array}$ & $\begin{array}{l}\text { Discussion articles, commentaries } \\
\text { or opinion pieces not presenting } \\
\text { empirical or theoretical research. } \\
\text { Grey literature if it did not have } \\
\text { details of authorship. }\end{array}$ \\
\hline
\end{tabular}

PCOS, Population Intervention Comparator Outcome Study Design.

literature was excluded if it did not meet the criteria for inclusion on date, format of reporting, type of data and details of authorship. Eligibility criteria are summarised in table 1.

\section{Data sources and search strategy}

We searched for empirical studies published between January 2006 and September 2016 on the following databases: PsychINFO, Ovid MEDLINE, Eric, Web of Science (Arts and Humanities Citation Index, Social Science and Science Citation Index), Scopus, PILOTS, CINAHL, SPORTDiscus and International Index to Performing Arts. There were no language restrictions.

Electronic databases were searched using a combination of Medical Subject Headings (MeSH) and free text terms. An example of the Ovid MEDLINE search strategy used can be found in online supplementary appendix 1 . All database searches were based on this strategy but were appropriately revised to suit each database.

Additionally, reference lists of all relevant reviews ${ }^{37-42}$ from the last 5 years were hand-searched to identify additional relevant empirical evidence. We also carried out a search for up-to-date UK unpublished (grey) literature completed between 2013 and 2016 via: (i) an online call for evidence on the What Works Centre for Wellbeing website between October and November 2016; (ii) contacting known experts in the field for recommendations of sport or dance sector reviews or repositories to search; (iii) a review of key sector websites; (iv) a search of the British Library EThOS website for unpublished $\mathrm{PhD}$ dissertations and (v) reviewing the titles of the first 100 results in a Google search with the use of key terms ('sport' AND 'physical activity' AND 'dance' AND 'wellbeing' AND 'young people'). 'Physical activity' was included as a search term because it is used by the sector when reporting on sport and dance activities.

\section{Study selection}

Two reviewers independently screened the titles and abstracts of all studies identified by the search strategy for their eligibility. Where it was not clear from the title and abstract whether a study was relevant, the selection criteria were independently applied to the full article to confirm its eligibility. Where two independent reviewers did not agree in their primary judgements they discussed the conflict and attempted to reach a consensus. If they could not agree then a third member of the review team considered the full paper and a majority decision was made. Online supplementary appendix 2 lists excluded studies and reasons for exclusions. 


\section{Data extraction}

Two review authors independently extracted data using a standardised form (online supplementary appendix 3). Discrepancies were resolved by discussion and consensus. Where agreement could not be reached, a third review author considered the paper and a majority decision was reached. The following data were extracted: (1) evaluation design and objectives (the interventions studied and control conditions used, including detail where available on the intervention content, dose and adherence, ethics); (2) sample (size, representativeness, reporting on dropout, attrition and details of participants including demographics and protected characteristics where reported); (3) the outcome measures (the scales used and the collection time-points, independence, validity, reliability, appropriateness to well-being impact questions); (4) analysis (assessment of methodological quality/limitations); (5) results and conclusions; (6) the presence of possible conflicts of interest for authors. In order to capture project details in the grey literature, we used an adapted version of the Public Health England Arts and Health Evaluation Framework ${ }^{43}$ and extracted project aims; costs; commissioners, partners and funding sources; intervention details; population and reported outcomes. Where available, evaluation details (aims, objectives, budget, procedures, timeline, data analysis and findings) were also extracted.

Our protocol included for us to contact the authors of articles if the required information could not be extracted and was essential for the interpretation of their results but we did not need to do this.

\section{Quality assessment}

To assess the methodological quality of the included published studies, two review authors independently applied the quality checklist for quantitative studies based on the Early Intervention Foundation checklist and detailed in the What Works Centre for Wellbeing methods guide ${ }^{44}$ (online supplementary appendix 4). The checklist was used to indicate if a specific study had been well designed, appropriately carried out and properly analysed. A summary of quality scores is presented in table 2 .

We then employed the Grading of Recommendations Assessment, Development and Evaluation working group methodology (GRADE) schema for judging strength and quality of evidence on well-being overall from sport and dance interventions. Four categories of evidence are used in GRADE; high, moderate, low or very low. Applying GRADE, randomised controlled trial (RCT) studies were initially judged as high quality and sound observational studies as low quality. Evidence was downgraded for methodological limitations, inconsistent findings, sparse data, indirect evidence and reporting bias. Evidence was graded upwards if there was a large magnitude of effect or a dose-response gradient. The PHE Arts for Health and Wellbeing Evaluation Framework ${ }^{43}$ was used to judge the quality of the grey literature in terms of the appropriateness of the evaluation design, the rigour of the data collection and analysis and precision of reporting.

\section{Data synthesis}

Due to heterogeneity of interventions and well-being outcomes between studies, a meta-analysis was not appropriate. We report the findings narratively. Summaries of the characteristics of the included studies were organised in a tabular form (table 3) and present information on the participants (number and characteristics), intervention and comparison conditions, outcomes and measure used, study design, conclusions and study limitations. Summaries of the results (number of participants, mean scores and SD) for each outcome measure at each measurement point, are presented in table 4 and synthesised in the text according to sport/dance intervention type and well-being outcomes. No studies reported CIs and so these have not been included.

\section{RESULTS}

\section{Search results}

After the removal of duplicates, the electronic searches returned 5597 records for title and abstract screening. Of these, 143 relevant articles remained for full-text assessment as well as 60 additional texts identified through other sources (6 through hand searching the reference lists of included reviews and 54 grey literature submissions were found: 12 received through the call for evidence, 33 via the extended search for grey literature and $9 \mathrm{PhDs}$ found on Ethos). After screening the 203 full texts, 11 studies were included in the systematic review. The search screening process is illustrated in figure 1 .

\section{Study characteristics}

The systematic review includes seven RCTs $^{45-51}$ (with a total of 884 participants) and one cohort study ${ }^{52}$ (93 participants) from the published literature. Three evaluation reports were included from the grey literature. A summary of the characteristics of the included papers is presented in table 3 . Table 4 provides a summary of the numerical results for each published study.

The included studies investigated the effects of a range of sport and dance interventions; the most common form of intervention reported were based on meditative practices including yoga ${ }^{46} 50$ and Baduanjin Qigong. ${ }^{48}$ Other interventions reported included body conditioning, aerobic exercise, ${ }^{47}$ dance forms delivered through dance training, ${ }^{45}$ hip-hop dance, ${ }^{47}$ an empowerment-based exercise intervention programme ${ }^{49}$ and specifically identified sports including, body conditioning, and ice skating ${ }^{47}$ and Nintendo Wii Active Games. ${ }^{51}$ Descriptions of interventions tended to be brief. All studies identified the frequency and type of intervention activity, the duration and content of activity sessions, the delivery site and the number of times per week participants took part. All differed in these characteristics as detailed in table 3 . Interventions in six of the RCT studies ${ }^{45-51}$ and 


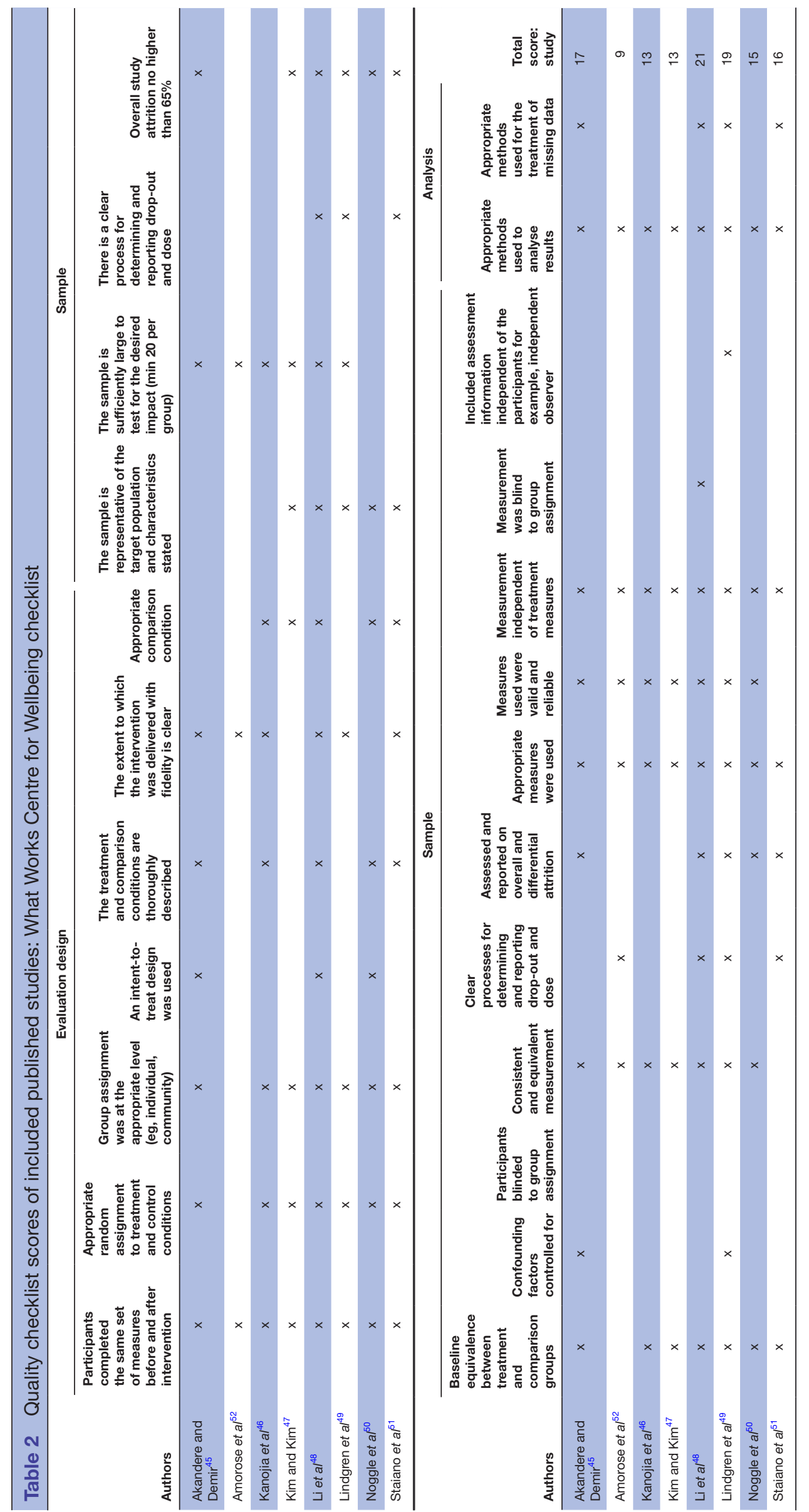

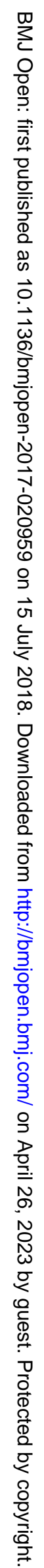




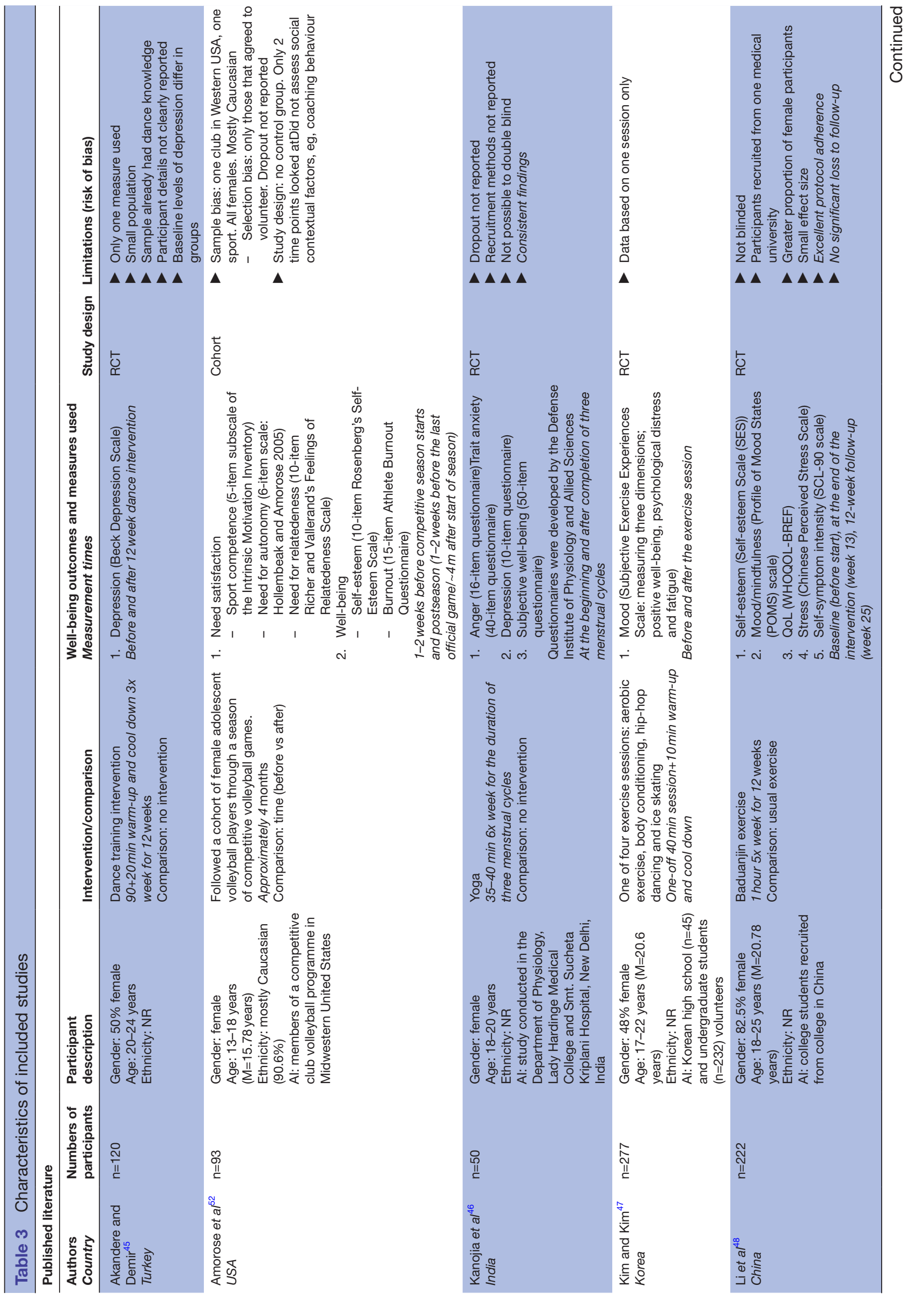




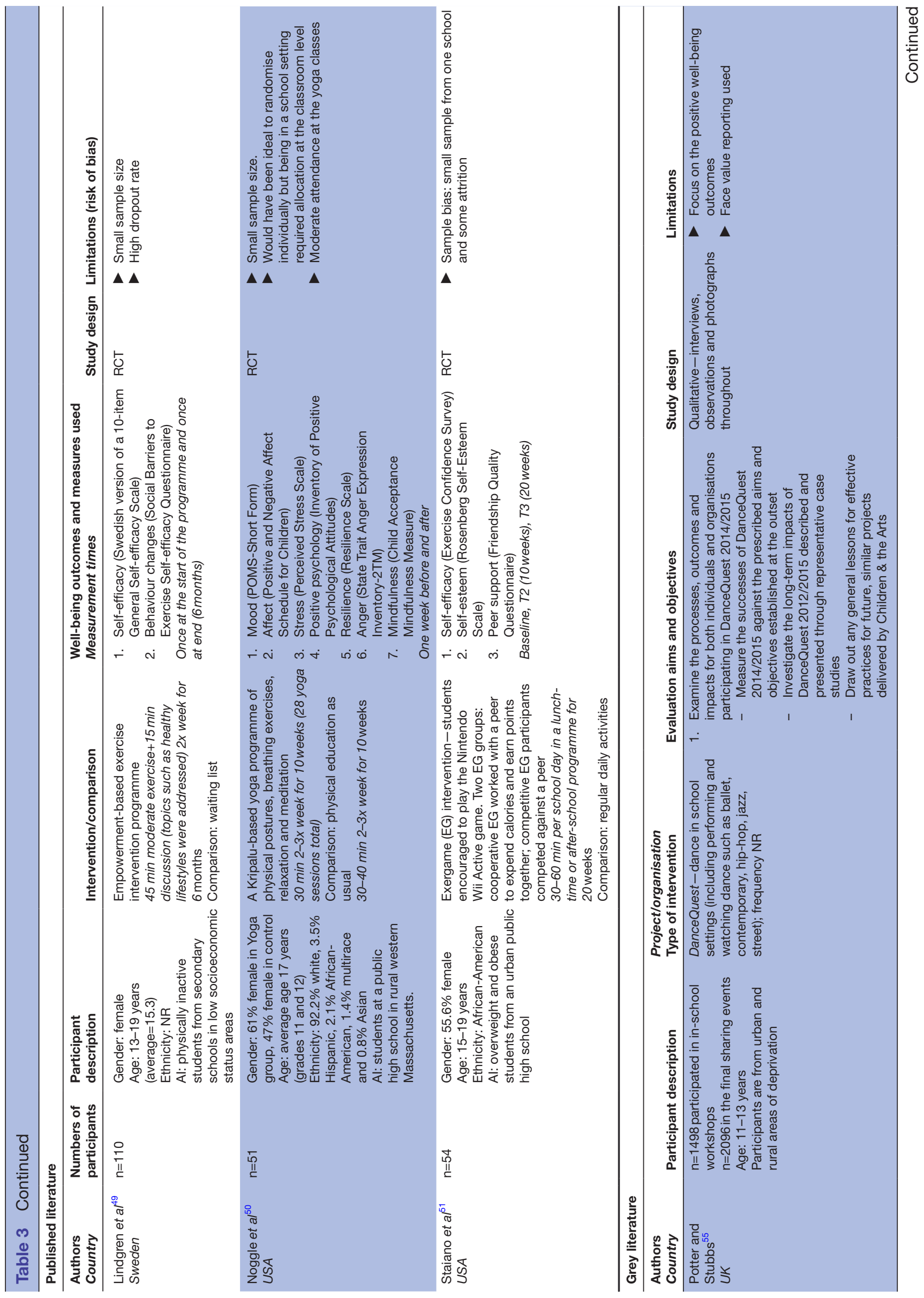

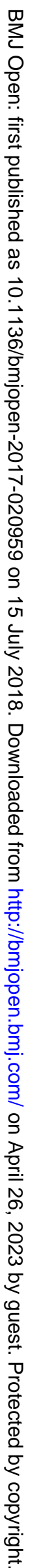




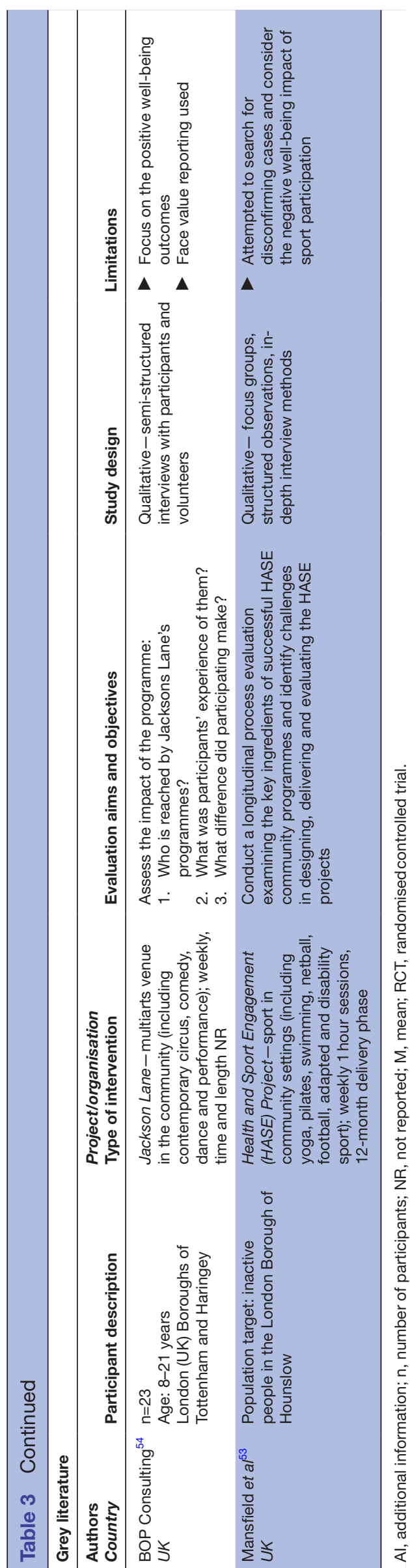

in the cohort study ${ }^{52}$ involved delivery by qualified sport or dance instructors in formal group sessions. One RCT used the Nintendo Wii Active Games Programme incorporating a cooperative or competitive peer-to-peer method of participation. ${ }^{51} \mathrm{~A}$ wide range of well-being measures were used and are summarised in online supplementary appendix 5.

Projects reported in the grey literature included the following interventions: martial arts, dance, gym-based exercise, exercise classes, swimming, netball, cycling and football, ${ }^{53}$ circus-based skills (eg, juggling, balancing, diabolo) ${ }^{54}$ and a range of dance forms. ${ }^{55}$ Interventions were led by instructors in group settings. Well-being was evaluated using descriptive statistics and/or thematic analysis from surveys, focus groups, interviews and structured observations.

All of the included studies were carried out in countries categorised in the same group as the UK in the OECD Development Assistance Committee (DAC) categories apart from two (one took place in India, ${ }^{46}$ and the other was based in $\operatorname{Korea}^{47}$ ). The sample participants in these two studies were university students, whose educational status indicates their relatively high socioeconomic status, making them broadly comparable with the categorisation of the DAC group in which the UK is located.

\section{Study quality}

The scores for the included studies from the What Works Centre for Wellbeing quality checklist for quantitative data are presented in table 2. The most frequent methodological weaknesses within the studies (with four or fewer studies meeting the criteria) were the absence of an intent-to-treat design, not having a clear process for determining and reporting dropout and dose, not having an appropriate method for the treatment of missing data, not controlling for confounding factors, not being able to blind participants or measurements and not including assessment information independent of the participants. Common (all studies meeting the criteria) strengths included using appropriate measures, independent of treatment measures, giving measures before and after the intervention/control and using appropriate methods to analyse the data. The results of the quality checklist varied across studies, with Amorose $e t a b^{52} 52$ scoring the worst (9 criteria met) and Li $e t a t^{48}{ }^{48}$ scoring the highest (21 criteria met).

The use of the GRADE schema for judging quality of evidence means that despite the predominance of RCT designs, overall the quality of the published evidence on sport and dance interventions to enhance well-being is low in respect of there being very little evidence in total, methodological limitations noted above, small sample sizes in studies and some sample bias.

Using the PHE Arts for Health and Wellbeing Evaluation Framework, the evidence from the grey literature were judged to have a high degree of credibility. The strongest reports included descriptive and theoretical detail about evaluation methods and acknowledged the 


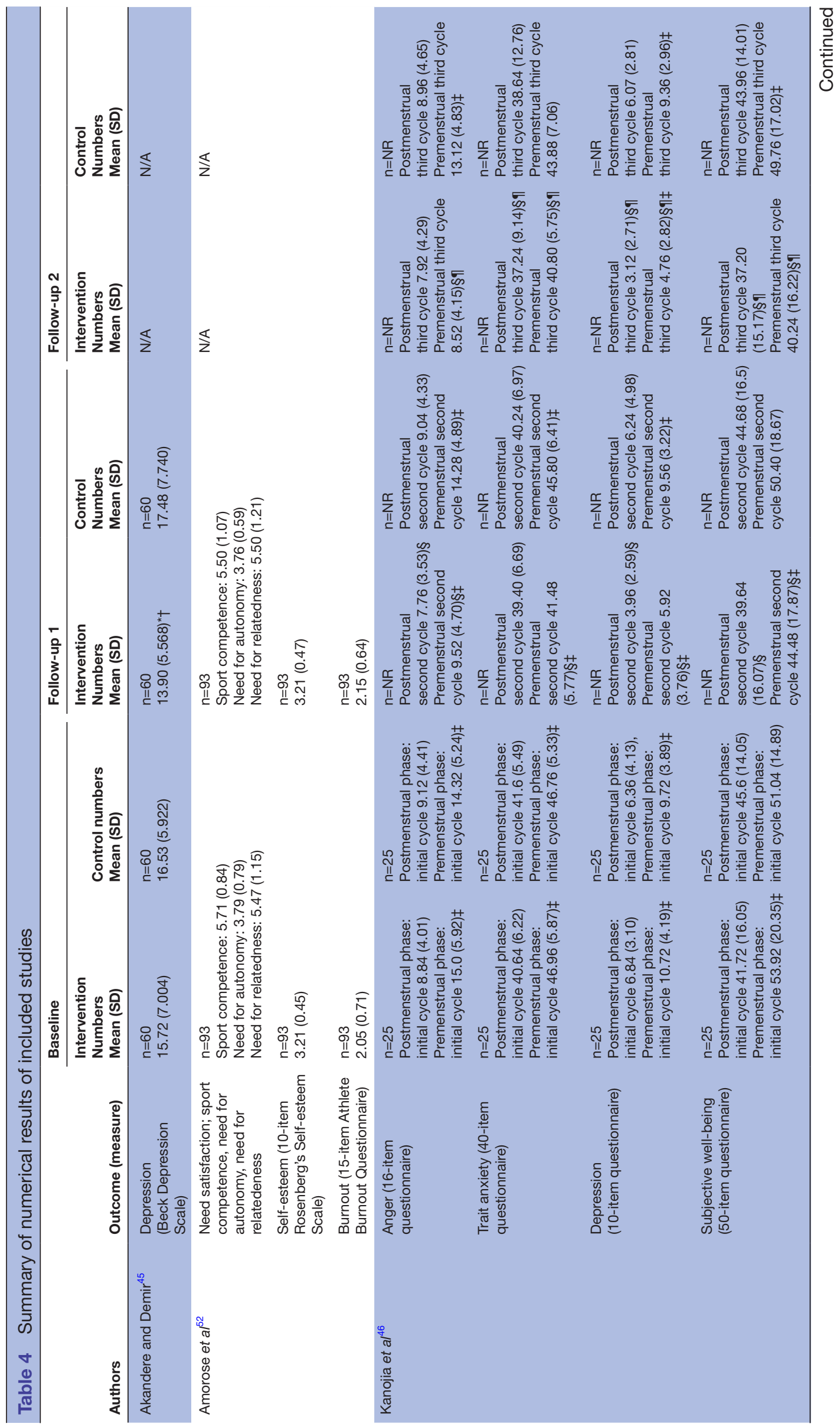

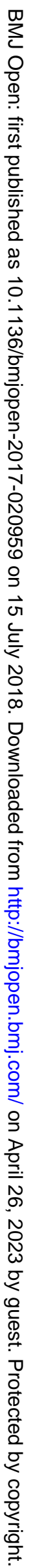




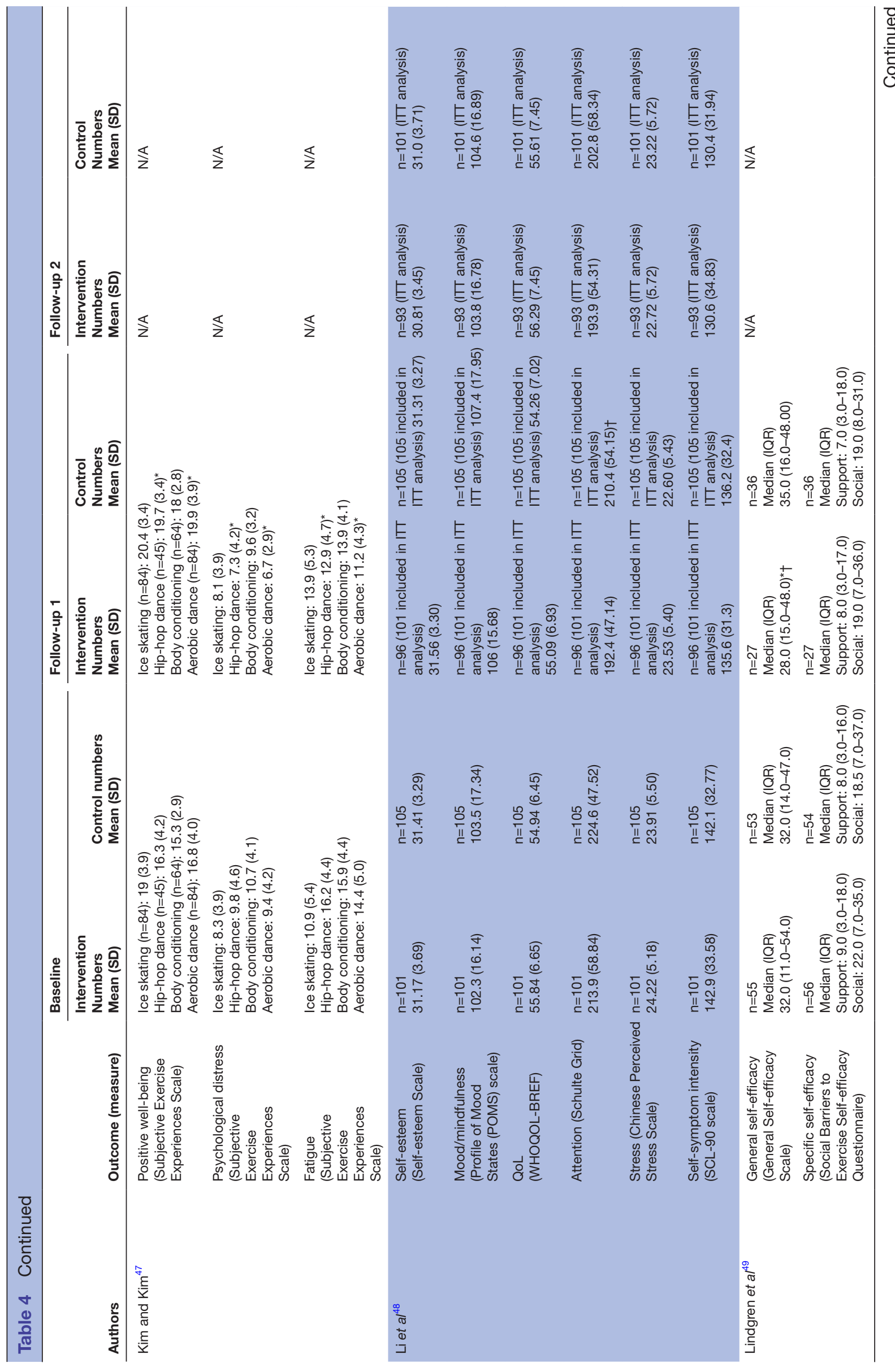

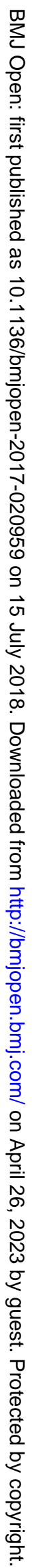




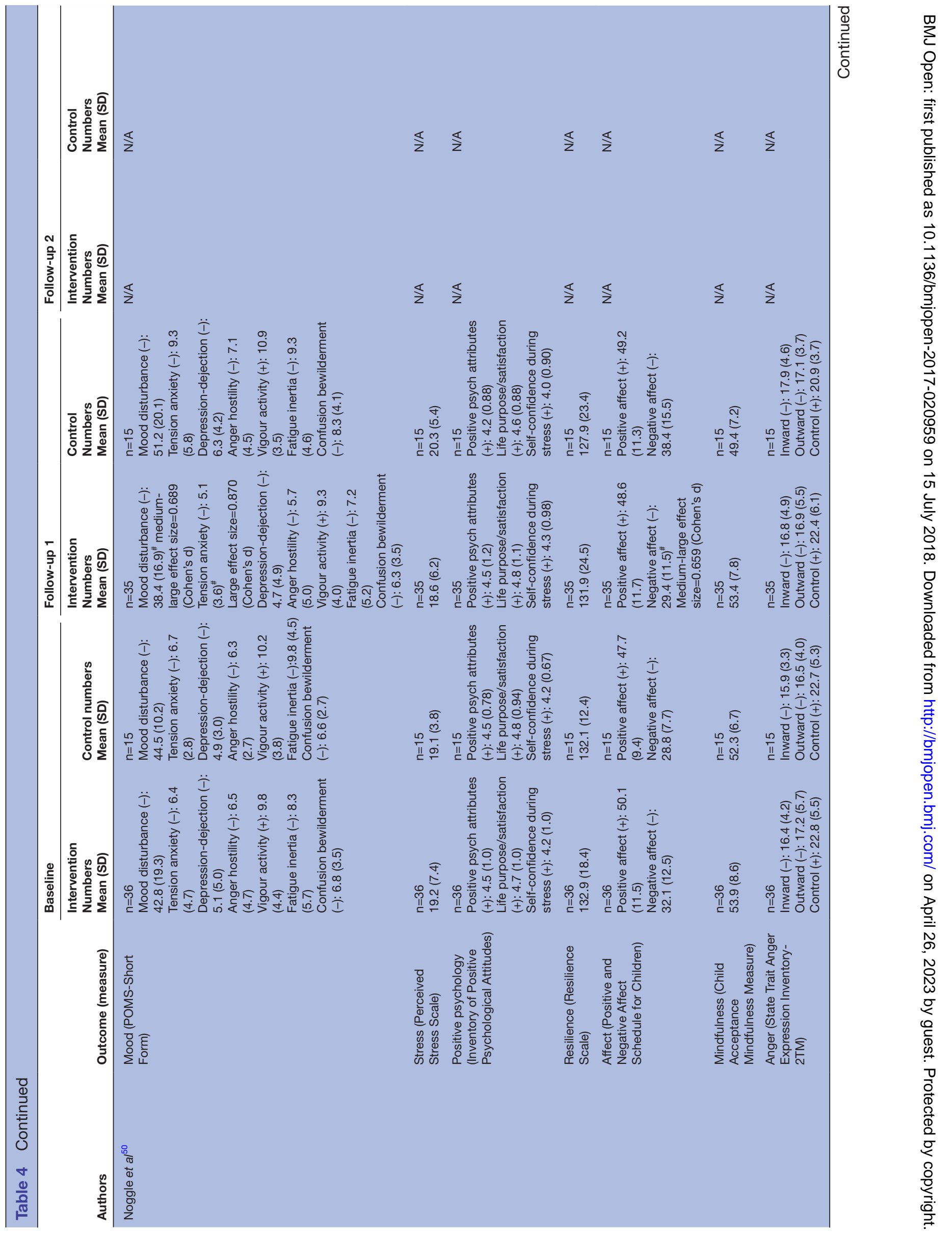




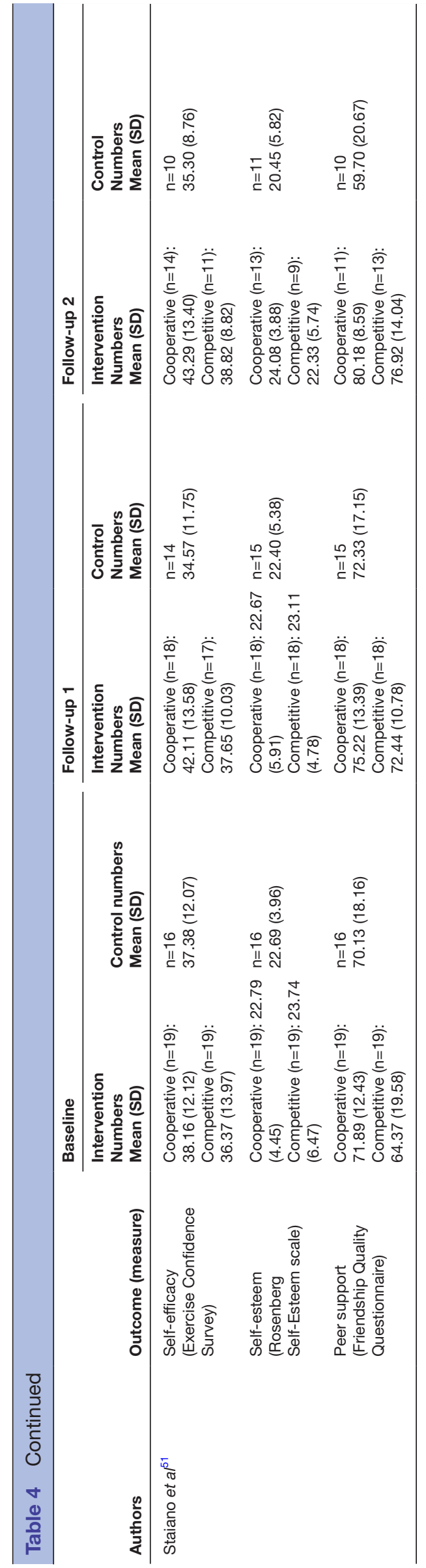

limitations of evaluation design. Two studies reported both preproject and postproject data. It was not always clear how themes were identified and developed and it was not always apparent that conclusions emerged from comprehensive data treatment. One report made a clear attempt to search for disconfirming cases and consider the negative well-being impact of sport participation, ${ }^{53}$ but evaluation reports tended to focus only on the positive impacts of sport and dance. Furthermore, there was a tendency in evaluations on dance and performance to rely on face value reporting of participants' accounts rather than developing latent forms of thematic analysis informed by identified theory where appropriate.

\section{The effect of meditative sport activity on well-being}

Three published RCT studies assessed the effectiveness of meditative practices including yoga ${ }^{46} 50$ and Baduanjin Qigong $^{48}$ on well-being in young people. All three studies used several different measures of well-being including mood scales for rating anger, anxiety, positive and negative affect, confusion/bewilderment and stress, anxiety and depression. ${ }^{46} 4850$ One study also included measures of self-esteem, quality of life, mindfulness and resilience. ${ }^{48}$ Two studies reported significantly improved well-being outcomes from taking part in yoga compared with a control group. ${ }^{46} 50$ One study found significant reductions between groups in total mood disturbance (medium-large effect size $=0.689$ (Cohen's $\mathrm{d}$ ), $\mathrm{p}=0.015$ ), tension and anxiety (large effect size $=0.870$ (Cohen's $\mathrm{d}), \mathrm{p}=0.002$ ) and negative affect (medium-large effect size $=0.659$ (Cohen's d), $\mathrm{p}=0.006) .{ }^{50}$ The second study found a significant reduction at 3 months compared with baseline in self-reported depression $($ effect size $=$ not reported (NR), postmenstrual phase $\mathrm{p}<0.001$, premenstrual phase $\mathrm{p}<0.001$ ), anxiety (effect size $=\mathrm{NR}$, postmenstrual $\mathrm{p}<0.05$, premenstrual $\mathrm{p}<0.001$ ), and anger (effect size $=\mathrm{NR}$, premenstrual $\mathrm{p}<0.001)$, as well as an improved overall sense of well-being (effect size $=\mathrm{NR}$, postmenstrual $\mathrm{p}<0.001$, premenstrual $\mathrm{p}<0.001) .{ }^{46}$ One study reported no significant difference in self-esteem, mindfulness, quality of life, stress or 'sympton' intensity in young people taking part in Baduanjin Qigong compared with usual exercise practice. ${ }^{48}$ No grey literature on yoga and well-being was included in this review.

\section{The effect of group/team sport on well-being}

Two published RCT studies ${ }^{49} 51$ and one cohort study ${ }^{52}$ examined the well-being outcomes of group sport activities. Two of these studies measured well-being using self-efficacy scales. ${ }^{49}{ }^{51}$ Two studies included a measure of self-esteem. ${ }^{51}{ }^{52}$ One study used a friendship quality assessment as a measure of well-being. ${ }^{51}$ One study measured well-being outcomes relating to need satisfaction theory (competence, autonomy and relatedness) ${ }^{52}$; an established approach to personal well-being research in sport psychology. The two studies using self-efficacy measures reported statistically significantly improved scores after taking part in group sport interventions compared with 


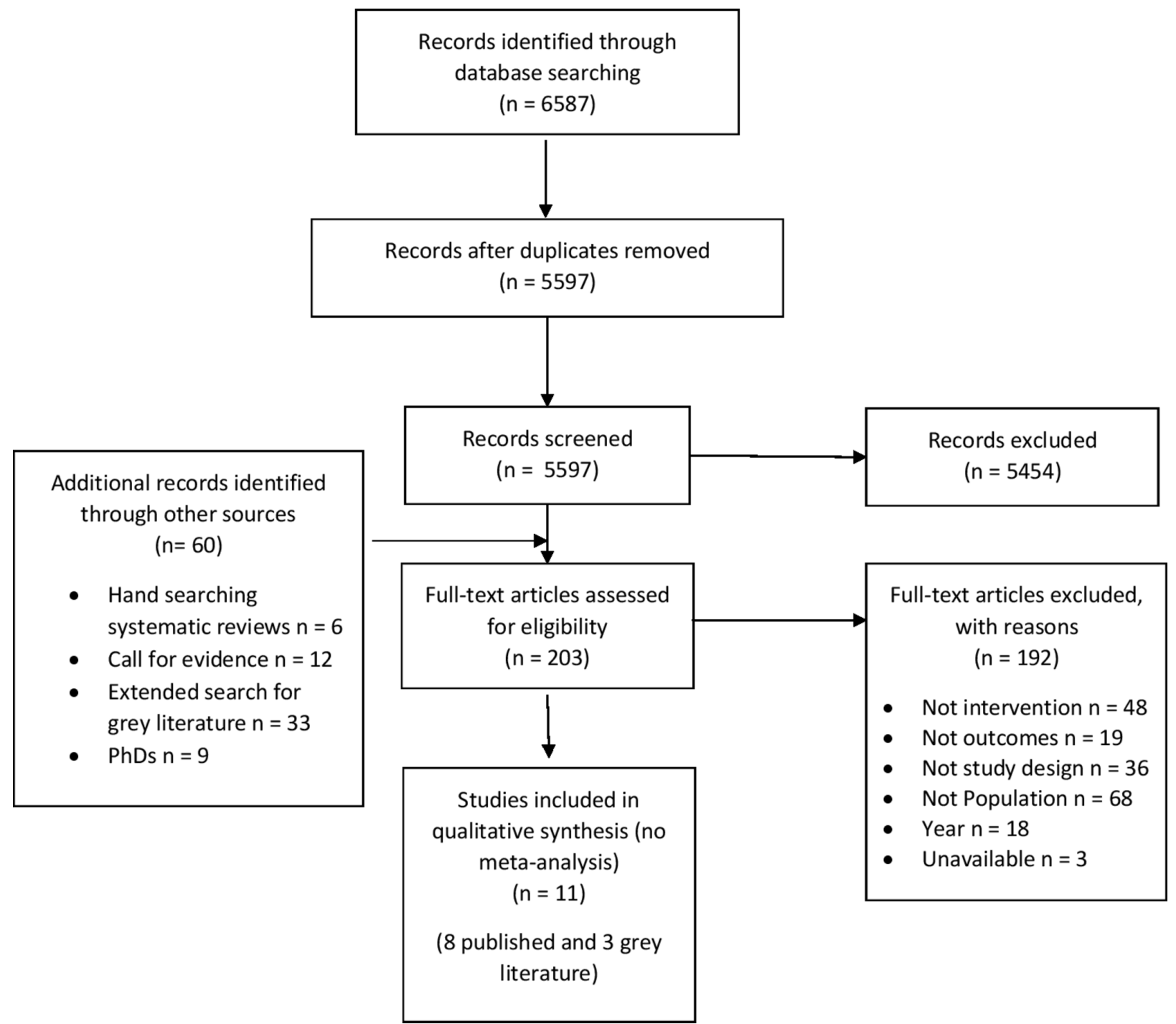

Figure 1 Preferred Reporting Items for Systematic Reviews and Meta-Analysis flow diagram of the search screening process.

the control (effect size $=\mathrm{NR}, \mathrm{p}=0.037^{49}$; cooperative condition $(\mathrm{M}=43.29, \mathrm{SD}=13.40)$ versus control group $(\mathrm{M}=35.30$, $\mathrm{SD}=8.76), \mathrm{t}=2.99, \mathrm{p}=0.005) .^{51}$

Both these studies employed interventions that were tailored to the needs of the participants and included elements of peer support. Significant increases in friendship quality were reported in taking part in sport compared with no sport (control condition: $\mathrm{M}=59.70$ $\mathrm{SD}=20.67$; cooperative condition: $\mathrm{M}=80.18$, SD-8.59, $\mathrm{t}=2.76, \quad \mathrm{p}=0.010 ; \quad$ competitive condition: $\mathrm{M}=76.92$, $\mathrm{SD}=14.04, \mathrm{t}=3.66, \mathrm{p}=0.001) .{ }^{51}$ No significant differences were reported for self-esteem scores between sport intervention groups compared with control. ${ }^{51}$ Changes in sports players' need to feel competent, autonomous and connected to others over the course of a sporting season were found to be positively related to changes in their overall sense of self-esteem. ${ }^{52}$ Qualitative findings from the one grey literature report identified negative and positive aspects of well-being associated with engagement in community sport including enhanced feelings of social connectedness, pleasure and sense of purpose as well as concerns related to personal capability, competence and unfavourable comparisons to peers who are 'sporty' ${ }^{53}$

The effect of group dance on well-being

Two published RCT studies examined the well-being outcomes (mood, fatigue scores and levels of depression) of group dance activities. ${ }^{45}$ One used a bespoke dance training programme, ${ }^{45}$ the other compared dance activities with sport and fitness activities. ${ }^{47}$ Taking part in dance exercise to music (aerobics) and hip-hop dancing aerobics were reported to significantly improve self-reported positive well-being and reduce distress and fatigue at the end of the intervention (effect size $=N R, p<0.05) .{ }^{47}$ Significant improvements on the self-reported Beck Depression Scale $(0-9=$ not depressed; $10-15=$ low-level depression; 16-23=medium-level depression, 24+=depressive) in participants not diagnosed with depression were reported from a dance training intervention $(\mathrm{M}=13.90, \mathrm{SD}=5.568)$ compared with control $(\mathrm{M}=17.48, \mathrm{SD}=7.740)$; $\mathrm{t}=2.911$, $\mathrm{p}=0.004 .{ }^{45}$ The grey literature reported questionnaire and interview results showing positive well-being associations 
from dance interventions in terms of increased confidence, sense of purpose and fun and exhilaration. ${ }^{54} 55$ Dance was also found to enhance, happiness, relaxation, playfulness, fun, social connectedness, aspiration, ambition and reduce isolation. ${ }^{54}$

\section{DISCUSSION}

\section{Principal findings and contribution to knowledge}

The relationship between organised physical activity and well-being in young people is not well understood. To our knowledge, this is the first systematic review of sport and dance interventions to promote subjective well-being in healthy young people (15-24 years). Overall, the published evidence suggests that meditative physical activity (examples included here were yoga and Baduanjin Qigong) has the potential to improve subjective well-being in terms of reduced anxiety, depression and anger, and enhanced positive mood in young people. This evidence also shows that taking part in dance can lead to positive well-being outcomes in terms of mood enhancement and self-reported reductions in feelings of depression in some youth populations. Unpublished grey literature illustrated that taking part in or watching dance, or other forms of performance-based physical activity and community sport can instil positive well-being feelings such as exhilaration and sense of purpose, and increased confidence, self-esteem and feelings of belonging and purpose. The findings support work that has associated physical activity with positive outcomes connected to depression, anxiety, self-esteem and cognitive function in children and adolescents. ${ }^{30} 5657$ The findings of this review also suggest that group-based sport and dance interventions may be important in ensuring positive well-being outcomes for young people taking part. Research supporting the physical and mental health contributions of physical activity has identified mediators such as organisational practices and the role of seeing other people who are similar to you becoming and being active, which are significant determinants of physical activity engagement. ${ }^{58}$ Our evidence also suggests that peer-supported delivery mechanisms in sport and dance programmes may support well-being enhancement for young people. This finding reinforces evidence-based calls for well-designed, clearly focused, expertly led, peer-peer youth interventions which incorporate high-quality peer leader training for positive well-being and mental health outcomes. ${ }^{59} 60$ The findings of the unpublished literature suggested that taking part in community sport is also associated with negative well-being connected to concerns about competency and capability. Several studies identify well-being-related and mental health risks in performance-based physical activity for young people including exercise addiction $^{6162}$ and disordered eating linked to feelings of inadequacy and self-criticism. ${ }^{63}$ Our findings support work that identifies the need to tailor physical activity interventions to the needs of those taking part in order to overcome negative perceptions of sport and barriers to involvement in order to maximise the potential for positive well-being outcomes from taking part. ${ }^{64}$

\section{Implications for policymakers and future research}

The findings reported in this review should be treated with caution because the quality of the published evidence on sport and dance interventions to enhance well-being is judged generally to be low. The evidence in this review is sparse, there are methodological limitations in the included studies and we still know very little about the effect of sport and dance interventions, which have the potential to influence the well-being of large numbers of people. No published UK studies were eligible for inclusion in this review. It is not possible to conclude that findings in this review are generalisable across countries or regionally in the UK. The lack of evidence identified in this review does not necessarily mean that well-being benefits are not accrued from taking part in sport and dance. Large-scale community sport and dance interventions have the potential to influence the well-being of people at population level. Recent national sport strategy in the $\mathrm{UK}^{45}$ identifies well-being as an outcome for sport and physical activity and needs to be accompanied by agreement about definitions and measures of well-being, a focus on measuring well-being outcomes and an emphasis on evaluating what works to enhance well-being in sport and dance. National agencies across the sport, culture and health sectors (eg, Department for Digital Culture Media and Sports (DCMS), Arts Counsil for England (ACE), Sport England, Public Health England (PHE) may be influential in promoting this approach; conversely, a lack of national lead may discourage academic and service delivery stakeholders from prioritising this.

Based on the evidence in this study, it is necessary to build evidence on well-being outcomes of sport and dance in healthy young people using agreed measures of well-being. There is a need for more well-designed, rigorous studies which are underpinned by relevant theory. Large-scale randomised controlled designs should be implemented in this target age group. Other rigorous and systematic study designs including evaluation of the complex community context and mechanisms of intervention effectiveness should be considered. The development of a multilevel programme of wellbeing evaluation training would support key policy and service delivery personnel and researchers in the sport and dance sectors in ensuring rigorous evaluation of interventions.

\section{CONCLUSION}

The evidence overall for the subjective well-being benefits of sport and dance interventions for healthy young people is limited in quality, selective and drawn from varied national and cultural contexts. The current state of the evidence means that it is not possible to identify 
a common effect of sport and dance on the subjective well-being of young healthy people or be certain about the influence of such physical activity on peoples' wellbeing. There are large gaps in our knowledge about the effect of sport and dance on the well-being of young people. Knowledge should be improved through rigorous complex community intervention research incorporating valid comparator groups to determine which sport and dance interventions are most effective in improving wellbeing in young people. Measurement of quantitative outcomes and evaluation of qualitative processes to determine how such interventions achieve their outcomes is needed.

Acknowledgements The authors acknowledge the expert support for the searches provided by library-based information scientists at Brunel University London and the University of Brighton.

Contributors The review was conceived and designed, and the protocol developed by LM, TK, CM, LGD, JL, AJ, ND, PD, ST, GJ, AP, AT and CV; article screening was carried out by LM, TK, AJ, LGD, JL and CV; data extraction, quality checks data interpretation were completed by LM, TK, AJ, LGD, JL and CV; and the manuscript drafted by LM and critically reviewed by TK, CM, LGD, JL, AJ, ND, PD, ST, GJ, AP, AT and CV.

Funding This study was funded by Economic and Social Research Council (ES/ N003721/1).

Competing interests None declared.

Patient consent Not required.

Provenance and peer review Not commissioned; externally peer reviewed.

Data sharing statement The appendix is available as online supplementary material and includes; Appendix 1, demonstration Ovid MEDLINE search strategy; Appendix 2, table of excluded studies; Appendix 3, the standardised data extraction form; Appendix 4, the What Works Centre for Wellbeing quality checklist (quantitative studies); Appendix 5, summary of SWB measures used in included studies.

Open access This is an open access article distributed in accordance with the terms of the Creative Commons Attribution (CC BY 4.0) license, which permits others to distribute, remix, adapt and build upon this work, for commercial use, provided the original work is properly cited. See: http://creativecommons.org/ licenses/by/4.0/

(C) Article author(s) (or their employer(s) unless otherwise stated in the text of the article) 2018. All rights reserved. No commercial use is permitted unless otherwise expressly granted.

\section{REFERENCES}

1. Huppert FA, So TT. Flourishing across Europe: application of a new conceptual framework for defining well-being. Soc Indic Res 2013;110:837-61.

2. Berry C. Wellbeing in four policy areas. Report by the all-party parliamentary group on wellbeing economics. London: Report, New Economics Foundation, 2014.

3. O'Donnell G, Deaton A, Durand M, et al. Wellbeing and Policy. London: Legatum Institute, 2014.

4. Department of Culture Media and Sport. Sporting future: a new strategy for an active nation. London: Crown Copyright, 2015.

5. Sport England. Towards an active nation strategy 2016-2021. London: DCMS, 2016.

6. One Dance UK. The UK body for dance. 2017 http://www. onedanceuk.org/

7. Start Active, Stay Active. A report on physical activity from the four home countries': Chief Medical Officers, 2011.

8. Dolan P. Happiness by design: change what you do, not how you think. London: Penguin, 2014.

9. Tinkler L, Hicks S. Measuring subjective well-being. London: Office for National Statistics, 2011:443-55.

10. Dolan P, METCALFE R. Measuring subjective wellbeing: recommendations on measures for use by national governments. J Soc Policy 2012;41:409-27.
11. Fujiwara D, Kudrna L, Dolan P. Quantifying and valuing the wellbeing impacts of culture and sport. London: DCMS, 2014. https://www.gov.uk/government/publications/quantifying-andvaluing-the-wellbeing-impacts-of-culture-and-sport. (accessed 7 Apr 2016).

12. Huppert FA. Challenges in Defining and Measuring Well-Being and Their Implications for Policy. Future directions in well-being: Springer International Publishing, 2017:163-7.

13. Huppert FA. Measurement really matters. Measuring wellbeing series; discussion paper 2: What Works Centre for Wellbeing, 2017.

14. Stewart-Brown S, Janmohamed K. Warwick-Edinburgh mental wellbeing scale: User guide, 2008.

15. Robins RW, Hendin HM, Trzesniewski KH. Measuring global selfesteem: construct validation of a single-item measure and the rosenberg self-esteem scale. Personality and Social Psychology Bulletin 2001;27:151-61.

16. Curran SL, Andrykowski MA, Studts JL. Short form of the profile of mood states (POMS-SF): psychometric information. Psychol Assess 1995;7:80-3.

17. What Works Centre for Wellbeing. credible evidence for better decisions to improve lives. https://www.whatworkswellbeing.org/

18. Daykin N, Mansfield L, Payne A, et al. What works for wellbeing in culture and sport? Report of a DELPHI process to support coproduction and establish principles and parameters of an evidence review. Perspect Public Health 2017;137:281-8.

19. Council of Europe. European Sports Charter. 1992 https://search. coe.int/cm/Pages/result_details.aspx?ObjectID=09000016804c9dbb

20. Daprati E, losa M, Haggard P. A dance to the music of time: aesthetically-relevant changes in body posture in performing art. PLoS One 2009;4:e5023.

21. Cavill N, Richardson D, Foster C. Improving health through participation in sport: a review of research and practice: BHF Health Promotion Research Groups, Oxford University and Sport England, 2012.

22. Oja P, Titze S, Bauman A, et al. Health benefits of cycling: a systematic review. Scand J Med Sci Sports 2011;21:496-509.

23. Oja P, Titze S, Kokko S, et al. Health benefits of different sport disciplines for adults: systematic review of observational and intervention studies with meta-analysis. $\mathrm{Br} J$ Sports Med 2015;49:434-40.

24. Mammen G, Faulkner G. Physical activity and the prevention of depression: a systematic review of prospective studies. Am J Prev Med 2013;45:649-57.

25. Ströhle A. Physical activity, exercise, depression and anxiety disorders. J Neural Transm 2009;116:777-84.

26. Robertson R, Robertson $A$, Jepson $R$, et al. Walking for depression or depressive symptoms: a systematic review and meta-analysis. Ment Health Phys Act 2012;5:66-75.

27. Bradt J, Shim M, Goodill SW. Dance/movement therapy for improving psychological and physical outcomes in cancer patients. Cochrane Database Syst Rev 2015;1:CD007103.

28. Ren J, Xia J. Cochrane Schizophrenia Group. Dance therapy for schizophrenia. Cochrane Database Syst Rev 2013;36.

29. Meekums B, Karkou V, Nelson EA. Cochrane Common Mental Disorders Group. Dance movement therapy for depression. Cochrane Database Syst Rev 2015;115:1-54.

30. Biddle SJ, Asare M. Physical activity and mental health in children and adolescents: a review of reviews. Br J Sports Med 2011;45:886-95.

31. Patel V, Flisher AJ, Hetrick S, et al. Mental health of young people: a global public-health challenge. Lancet 2007;369:1302-13.

32. Kieling $\mathrm{C}$, Baker-Henningham $\mathrm{H}$, Belfer $\mathrm{M}$, et al. Child and adolescent mental health worldwide: evidence for action. Lancet 2011;378:1515-25.

33. McGorry PD, Goldstone SD, Parker AG, et al. Cultures for menta health care of young people: an Australian blueprint for reform. Lancet Psychiatry 2014;1:559-68.

34. Moher D, Liberati A, Tetzlaff J, et al. Preferred reporting items for systematic reviews and meta-analyses: the PRISMA statement. Int J Surg 2010;8:336-41.

35. Ogilvie D, Foster CE, Rothnie $\mathrm{H}$, et al. Interventions to promote walking: systematic review. BMJ 2007;334:1204.

36. Ogilvie D, Egan M, Hamilton V, et al. Promoting walking and cycling as an alternative to using cars: systematic review. BMJ 2004;329:763.

37. Burkhardt J, Brennan C. The effects of recreational dance interventions on the health and well-being of children and young people: A systematic review. Arts Health 2012;4:148-61.

38. Koch S, Kunz T, Lykou S, et al. Effects of dance movement therapy and dance on health-related psychological outcomes: a metaanalysis. Arts Psychother 2014;41:46-64. 
39. Korber K. Quality assessment of economic evaluations of health promotion programs for children and adolescents-a systematic review using the example of physical activity. Health Econ Rev 2015;5:35.

40. Liu M, Wu L, Ming Q. How does physical activity intervention improve self-esteem and self-concept in children and adolescents? Evidence from a meta-analysis. PLoS One 2015;10:e0134804.

41. Rössler R, Donath L, Verhagen E, et al. Exercise-based injury prevention in child and adolescent sport: a systematic review and meta-analysis. Sports Med 2014;44:1733-48.

42. Thompson Coon J, Boddy K, Stein K, et al. Does participating in physical activity in outdoor natural environments have a greater effect on physical and mental wellbeing than physical activity indoors? A systematic review. Environ Sci Technol 2011;45:1761-72.

43. Daykin N, Joss T. GOV.UK. Public health England arts and health evaluation framework. 2016 https://www.gov.uk/government/ publications/arts-for-health-and-wellbeing-an-evaluation-framework

44. Snape D, Meads C, Bagnall A, et al. A guide to our evidence review methods. 2017 https://whatworkswellbeing.org/product/a-guide-toour-evidence-review-methods/

45. Akandere M, Demir B. The effect of dance over depression. Coll Antropol 2011;35:651-6.

46. Kanojia S, Sharma VK, Gandhi A, et al. Effect of yoga on autonomic functions and psychological status during both phases of menstrual cycle in young healthy females. J Clin Diagn Res 2013;7:2133-9.

47. Kim S, Kim J. Mood after various brief exercise and sport modes: aerobics, hip-hop dancing, ice skating, and body conditioning. Percept Mot Skills 2007;104:1265-70.

48. Li M, Fang Q, Li J, et al. The effect of chinese traditional exercise-baduanjin on physical and psychological well-being of college students: a randomized controlled trial. PLoS One 2015;10:e0130544.

49. Lindgren E-C, Baigi A, Apitzsch E, et al. Impact of a six-month empowerment-based exercise intervention programme in non-physically active adolescent Swedish girls. Health Educ J 2011;70:9-20.

50. Noggle JJ, Steiner NJ, Minami T, et al. Benefits of yoga for psychosocial well-being in a US high school curriculum: a preliminary randomized controlled trial. J Dev Behav Pediatr 2012;33:193-201.

51. Staiano AE, Abraham AA, Calvert SL. Adolescent exergame play for weight loss and psychosocial improvement: a controlled physical activity intervention. Obesity 2013;21:598-601.
52. Amorose AJ, Anderson-Butcher D, Cooper J. Predicting changes in athletes' well being from changes in need satisfaction over the course of a competitive season. Res $Q$ Exerc Sport 2009;80:386-92

53. Mansfield L, Kay T, Anokye N, et al. Evaluation of the Design and Delivery of the HASE project. The health and sport engagement (HASE) intervention and evaluation project: the design, delivery and evaluation of a complex community sport intervention for improving physical activity, health and wellbeing. 1, 2017.

54. BOP Consulting. The social impact of Jacksons Lane. 2016 https:// jacksonslane.rooftop.io/sites/18/2016/10/05112305/JL-SocialImpact-Study.pdf

55. Potter S, Stubbs FP. Dance Quest 2012-15 Evaluation Report. 2015.

56. Brown HE, Pearson N, Braithwaite RE, et al. Physical activity interventions and depression in children and adolescents. Sports Medicine 2013;43:195-206.

57. Badura P, Geckova AM, Sigmundova D, et al. When children play, they feel better: organized activity participation and health in adolescents. BMC Public Health 2015;15:1090.

58. Bauman AE, Reis RS, Sallis JF, et al. Correlates of physical activity: why are some people physically active and others not? Lancet 2012;380:258-71.

59. Coleman N, Sykes W, Groom C. Peer support and children and young people's mental health Research review Crown Copyright Independent Social Research (ISR), Department of Education. 2017 https://www.gov.uk/government/uploads/system/uploads/ attachment_data/file/603107/Children_and_young_people_s_mental_ health_peer_support.pdf

60. Repper J, Carter T. A review of the literature on peer support in mental health services. J Ment Health 2011;20:392-411.

61. Landolfi E. Exercise addiction. Sports Med 2013;43:111-9.

62. Villella C, Martinotti G, Di Nicola M, et al. Behavioural addictions in adolescents and young adults: results from a prevalence study. J Gambl Stud 2011;27:203-14.

63. Meyer C, Taranis L, Goodwin H, et al. Compulsive exercise and eating disorders. Eur Eat Disord Rev 2011;19:174-89.

64. Rosso E, McGrath R. Promoting physical activity among children and youth in disadvantaged South Australian CALD communities through alternative community sport opportunities. Health Promot J Austr 2016;27:105-10. 